\title{
Uncovering the Connection Between Low-Frequency Dynamics and Phase Transformation Phenomena in Molecular Solids
}

\author{
Michael T. Ruggiero, ${ }^{1,2, *}$ Wei Zhang, ${ }^{3}$ Andrew D. Bond, ${ }^{4}$ Daniel M. Mittleman, ${ }^{3}$ and J. Axel Zeitler ${ }^{1}$ \\ ${ }^{1}$ Department of Chemical Engineering and Biotechnology, University of Cambridge, \\ Philippa Fawcett Drive, Cambridge, CB3 0AS, United Kingdom \\ ${ }^{2}$ Present Address: Department of Chemistry, University of Vermont, \\ 82 University Place, Burlington, VT 05405, United States of America \\ ${ }^{3}$ School of Engineering, Brown University, Providence, RI, 02912, United States of America \\ ${ }^{4}$ Department of Chemistry, University of Cambridge, \\ Lensfield Road, Cambridge, CB2 1EW, United Kingdom
}

(Dated: April 9, 2018)

\begin{abstract}
The low-frequency motions of molecules in the condensed phase have been shown to be vital to a large number of physical properties and processes. However, in the case of disordered systems it is often difficult to elucidate the atomic-level details surrounding these phenomena. In this work, we have performed an extensive experimental and computational study on the molecular solid camphor, which exhibits a rich and complex structure-dynamics relationship, and undergoes a order-disorder transition near ambient conditions. The combination of X-ray diffraction, variable temperature and pressure terahertz time-domain spectroscopy, ab initio molecular dynamics, and periodic density functional theory calculations enables a complete picture of the phase transition to be obtained, inclusive of mechanistic, structural and thermodynamic phenomena. Additionally, the low-frequency vibrations of a disordered solid are characterised for the first time with atomic-level precision, uncovering a clear link between such motions and the phase transformation. Overall, this combination of methods allows for significant details to be obtained for disordered solids and the associated transformations, providing a framework that can be directly applied for a wide range of similar systems.
\end{abstract}

Low-frequency (terahertz) vibrational dynamics have been shown to be one of the major factors that dictate the properties of condensed phase materials, ranging from thermodynamic to mechanical phenomena.[1, 2] Additionally, a number of recent investigations have suggested that terahertz motions, which often involve large amplitude displacements of entire molecules, play a vital role in the proper functioning of materials. [3-8] However, the dependence of the low-frequency vibrations on both the intramolecular and intermolecular potential energy hypersurfaces leads to unique spectral fingerprints, which cannot be interpreted without additional analysis.[9] Over the past decade, computational tools have enabled much better insight into the origins of the terahertz response of crystalline solids.[10] In contrast, the interpretation of the response of disordered materials at terahertz frequencies remains a topic of great interest.[11]

Disordered materials make up a large portion of condensed phase systems, with many biological, pharmaceutical, and semiconducting technologies all based on the lack of any long-range order.[12-14] Given the prevalence of disordered materials, there exists a clear need for an appropriate method to gain atomic-level insight into the vibrational dynamics and associated effects. However, the difficulty in acquiring these, both experimentally and theoretically, have hindered progress.[15] In addition, it has been suggested that phase transformations in the condensed phase, e.g. crystalline polymorphism, are also strongly linked to the terahertz dynamics, with previous studies suggesting that particular vibrational motions can be directly correlated with the transformation mechanisms themselves.[16] But again, the lack of any direct experimental measurement of such transformation intermediates makes understanding these mechanisms difficult without a large degree of speculation.

Here, we use a combination of X-ray diffraction, temperature and pressure-dependent terahertz time-domain spectroscopy (THz-TDS), periodic density functional theory (DFT), and ab initio molecular dynamics (AIMD) simulations to fully describe the order-disorder phase transition in a well-studied molecular solid, homochiral R-camphor. The combination of these techniques makes it possible to uncover a complete picture of the lowfrequency dynamics in both the ordered crystalline and orientationally disordered phases of camphor, in addition to obtaining the bulk packing structure of the disordered phase for the first time.

Solid camphor exhibits rich structural and dynamic flexibility, as it can form an ordered crystal at lowtemperature $(<244 \mathrm{~K})$, and two disordered phases at higher-temperatures.[17] While the higher-temperature phases of camphor exhibit orientational disorder, the system still retains a degree of order, making it a valuable solid for understanding the mechanisms and dynamics associated with solid-state phase transitions.[18] The lowtemperature (LT) phase of camphor has been extensively characterised, $[19,20]$ however, there is a corresponding dearth of information related to the high-temperature phases of camphor. As previously shown, the THz-TDS spectrum of the LT and first high-temperature (HT) 
forms of camphor are substantially different, with the LT-form exhibiting a large number of observable features at terahertz frequencies $(0.3-3 \mathrm{THz})$, while the HT-form does not contain any well resolved absorption peaks.[20, 21]

In order to study the phase-transition in solid camphor, AIMD simulations were performed with the fully-periodic CP2K software package.[22, 23] All simulations were performed with the Grimme-D3 dispersion corrected[24, 25] PBE density functional,[26] and the atomic orbitals were represented using the double- $\zeta$ DZVP basis set[27] and Goedecker-Teter-Hutter (GTH) pseudopotentials.[28] Calculations were performed within both the canonical (NVT) and isothermalisobaric (NPT) ensembles, making use of a Nose-Hoover chain thermostat[29] and an extended Nose-Hoover barostat for pressure.[30, 31]

Initially, NPT simulations were performed on the previously solved LT-structure.[19] A supercell containing 4 unit cells (32 molecules, 864 atoms total) was generated, and the fully-periodic (translational symmetry in all three Cartesian axes) simulations were set to a temperature of $325 \mathrm{~K}$ (above the transition temperature of $244 \mathrm{~K}$ ) with a time step of $1 \mathrm{fs}$. The simulation box size was set sufficiently larger than what was previously determined to be suitable for fully disordered systems in order to minimize spurious interactions between molecules that are generated by translational symmetry.[32] Immediately the camphor units begin to rotate with respect to each other, breaking space-group and translational symmetry. In addition, throughout the first $25 \mathrm{ps}$, the individual rows of camphor molecules slide past one another, resulting in a marked change in one of the simulation cell angles from $90^{\circ}$ to $120^{\circ}$. After approximately $25 \mathrm{ps,}$ the cell stabilises and there is no further translational diffusion, but the molecules continue to undergo reorientational Brownian motions in their sites. Long-range order (i.e. the rows of camphor molecules) is not significantly distorted throughout the course of the simulation, maintaining a partially-ordered character, and was not due to the imposed periodicity as shown through simulations on a non-periodic structure that yielded similar results.

Throughout the simulation there are a significant number of transient localisation events, with at least a pair of molecules (and occasionally more) orienting themselves so that their molecular dipole-moments are favourably arranged. These were used as the basis for extracting a single-crystalline unit cell from the partially disordered simulation by fitting a box around a pair of molecules and imposing translational symmetry, as shown in Figure 1. The generated single crystal was optimised within a hexagonal Bravais lattice (to mimic the supercell angles) using the CRYSTAL17 DFT software package.[33] As with the AIMD simulation, the D3-corrected[24, 25] $\mathrm{PBE}[26]$ density functional was used, along with the double- $\zeta$ def2-SVP basis set.[34] The resulting structure yielded a cell with lattice parameters of $a=b=$ $6.943 \AA, c=11.209 \AA, \alpha=\beta=90^{\circ}$, and $\gamma=120^{\circ}$, with $Z=2$. From this structure, a simulated powder Xray diffraction (PXRD) pattern was generated and found to be in good agreement with the experimental pattern, however slightly overestimated due to the DFT-optimised structure being a $0 \mathrm{~K}$ crystal. Therefore, NPT simulations were performed at $300 \mathrm{~K}$ starting from the optimised coordinates, and upon equilibration the simulated unit cell lengths were $a=b=7.073 \AA$ and $c=11.486 \AA$, in excellent agreement with the experiment (see Figure 1).

Any attempt to allow the newly-determined structure of the HT-form to optimise without hexagonal symmetry constraints resulted in the structure reversing to the LT-form, which also occurs when low-temperature AIMD simulations were performed, albeit at a much slower rate (see Figure 1). This indicates that the dynamics of the system, specifically the low-frequency dynamics (since they are activated at relatively ambient conditions), are of critical importance for understanding the origins of the phase-transition mechanism.

The experimental temperature and pressuredependent THz-TDS spectra of camphor were obtained using a custom-built THz-TDS spectrometer (see Supporting Information).[21] The THz-TDS spectrum of the LT-phase contains a number of resolvable features, while the spectrum of the HT-phase is essentially featureless, a common trait of disordered solids.[35, 36] While the LT-phase has had its spectrum assigned previously,[20] the spectrum of the HT-phase (and in fact, the low-frequency vibrational spectra of disordered solids in general) has not been quantitively assigned with quantum-mechanical accuracy and atomic-level precision to date.

The vibrational dynamics of both phases of camphor were simulated by both the AIMD and static-DFT techniques. For the former case, NVT simulations were performed on a single unit cell of the LT-form (at $100 \mathrm{~K}$ ) and a supercell of the HT-form (at $300 \mathrm{~K}$ ), each containing 8 camphor molecules (216 atoms), using previously described methodologies.[37, 38] The results, shown in Figure 2, show an good agreement with experiment for both phases, both in regard to frequency position and intensity, yet the experimental results are not able to fully resolve the lowest-frequency features due to limitations of the reflection geometry used.[20]

Analysis of the AIMD trajectories shows that while the LT-form exhibits the typical crystalline-behaviour of the individual molecules oscillating about their potential energy minima, the molecules in the HT-form are almost instantly decoupled from one another and move freely within their crystalline sites. As with the NPTsimulations, there does exist a large number of transient ordering events. However, the thermal activation of 

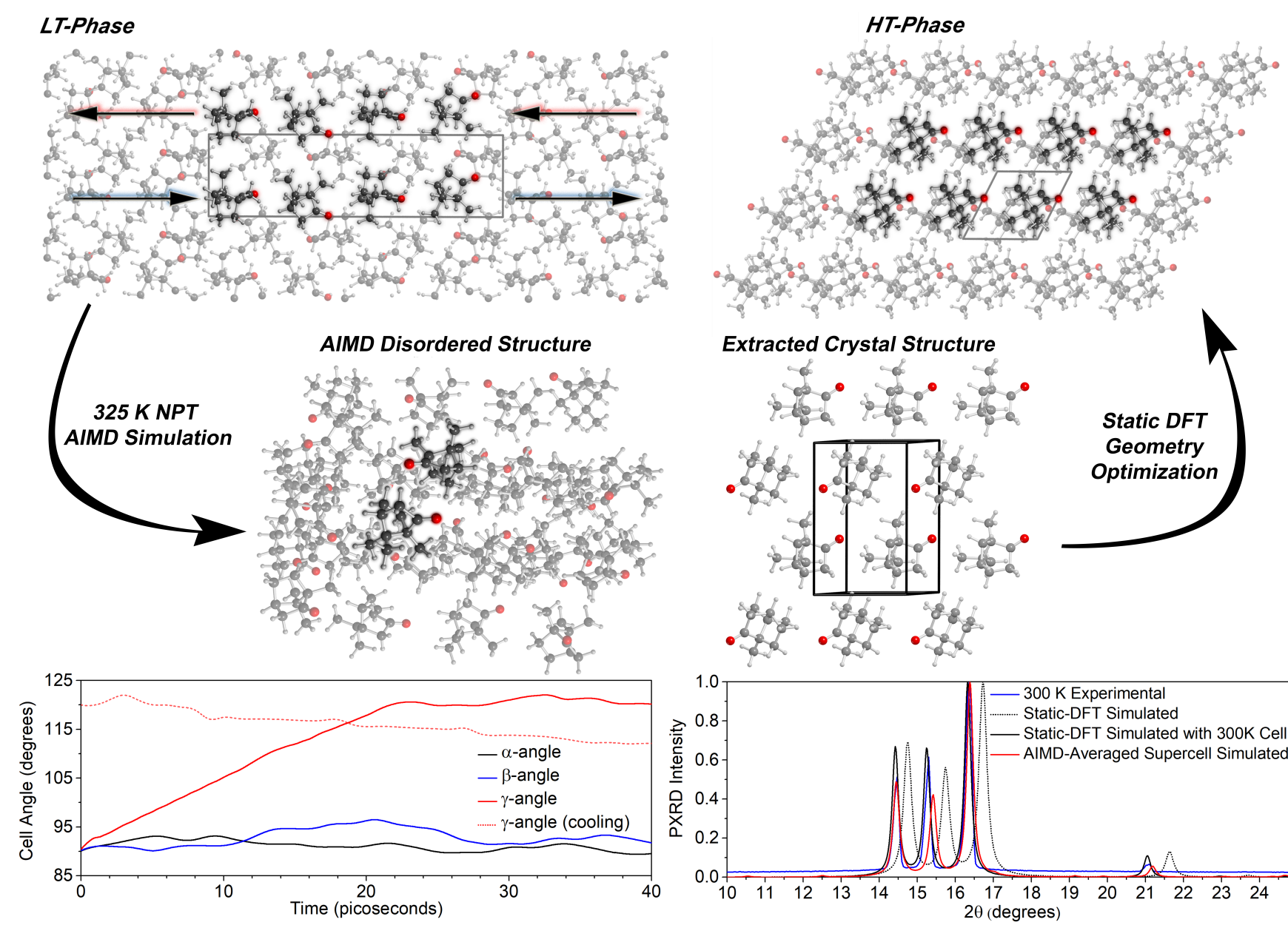

Extracted Crystal Structure
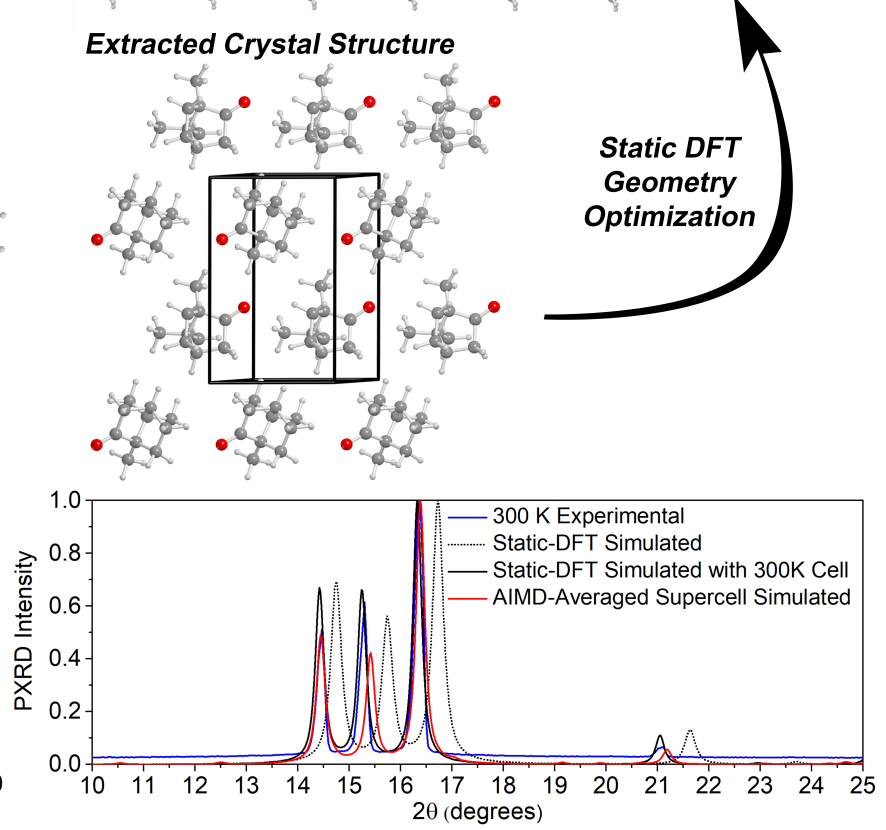

FIG. 1: Experimental structure of the LT and HT-phases of camphor (arrows represent row diffusion and the predicted $1.69 \mathrm{THz}$ vibrational mode, details in text), and the structure after the AIMD-NPT equilibration. The dimer pair used to generate the crystallographic structure of the HT-phase is darkened. The bottom left panel shows the NPT-supercell angles as a function of time, with equilibrium of the HT-phase achieved after 25 ps. The dotted curve is the $\gamma$-angle starting from the disordered structure and running a NPT simulation at $100 \mathrm{~K}$. The bottom right panel is the comparison between the experimental PXRD pattern (blue) and the patterns generated from the static-DFT simulated structure (dotted black), static-DFT optimized structure within $300 \mathrm{~K}$ NPT-determined cell vectors (black), and AIMD NVT-averaged pattern (red).

the low-amplitude vibrations clearly is enough to overcome the intermolecular potential energy barriers, although to a minor degree compared to fully-disordered solids (which typically exhibit large-scale diffusive-type motions).[32] Moreover, the weakened intermolecular interactions are evident in the red-shifting of the HTspectrum as compared to the LT-spectrum, as the smaller vibrational force constants reduce the energy of the individual vibrations. Supporting this is the calculated cohesive energy of an individual camphor molecule in the two crystals, with the HT-form exhibiting approximately $33 \%$ less binding energy than the LT-form.

Vibrational simulations were performed within the harmonic approximation using CRYSTAL17.[39, 40] The results of the static-DFT simulations are provided in Fig- ure 3a (LT-results are provided in the Supporting Information). The predicted spectrum, which has been convolved using Lorentzian lineshapes, generally reproduces the experimental spectrum above $1.2 \mathrm{THz}$, but a noticeable lack of agreement exists below that frequency. Visualisation of the normal modes reveals that, as is the case of the LT-phase, the sub $3 \mathrm{THz}$ motions are primarily hindered-rotational motions of the individual camphor molecules, with the exception of a mode predicted at $1.69 \mathrm{THz}$, which corresponds to the row diffusion described in Figure 1. In the HT-form, the lowest frequency mode $(0.503 \mathrm{THz})$ contains a translational component, which directly corresponds to the phasetransition pathway as determined from the AIMD simulations. The predicted features at 1.077 and $1.510 \mathrm{THz}$ 


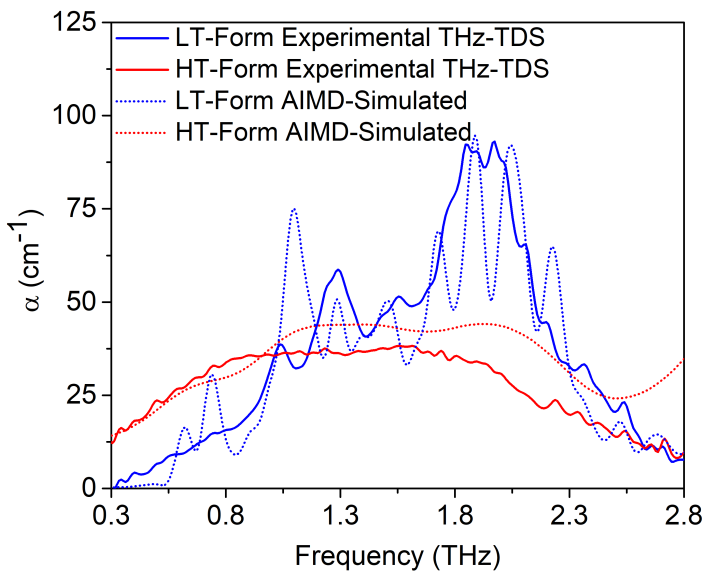

FIG. 2: Experimental (solid lines) and AIMD-simulated (dotted lines) THz-TDS spectra of the HT-form (red) and LT-form (blue) of camphor.

are coupled librations of the two camphor molecules in the unit cell, representing in-phase and out-of-phase hindered-rotations, respectively. The final three predicted features at $1.830,2.039$, and $2.197 \mathrm{THz}$ are all uncoupled librations of an individual camphor molecule in the unit-cell, with only one of the two molecules rotating significantly. Above $3 \mathrm{THz}$, the modes show little intermolecular character and consist mainly of internal torsions, such as methyl-rotation modes at $5.7 \mathrm{THz}$, demonstrating the importance of the $0.3-3 \mathrm{THz}$ region of studying intermolecular effects.

The combination of specific atomic-level vibrational information from the static-DFT calculations and the temperature and disorder-dependent AIMD simulations enables the origins of the differences between the two different simulated spectra to be determined. Using the DFT-predicted vibrational normal mode frequency positions, four Lorentzian curves were fit to the AIMD spectrum, resulting in excellent agreement as shown in Figure 3b. It is important to note that because of the similarity of the three modes between 1.8 and $2.2 \mathrm{THz}$, one Lorentzian was used to represent all three vibrations. The lack of intensity for the DFT-calculated lowerfrequency modes arises due to the imposed crystallographic symmetry. However, in the disordered solid, no such symmetry constraint exists, resulting in a larger net change in dipole-moment of the solid, leading to the increased low-frequency intensity that is observed. In the case of the higher-frequency modes, because they represent uncoupled motions of individual molecules, their predicted intensities are similar in both the crystalline and disordered states. Finally, while most of the modes shift to lower-energy from the simulated static-DFT to AIMD spectra, the lowest-frequency vibration actually
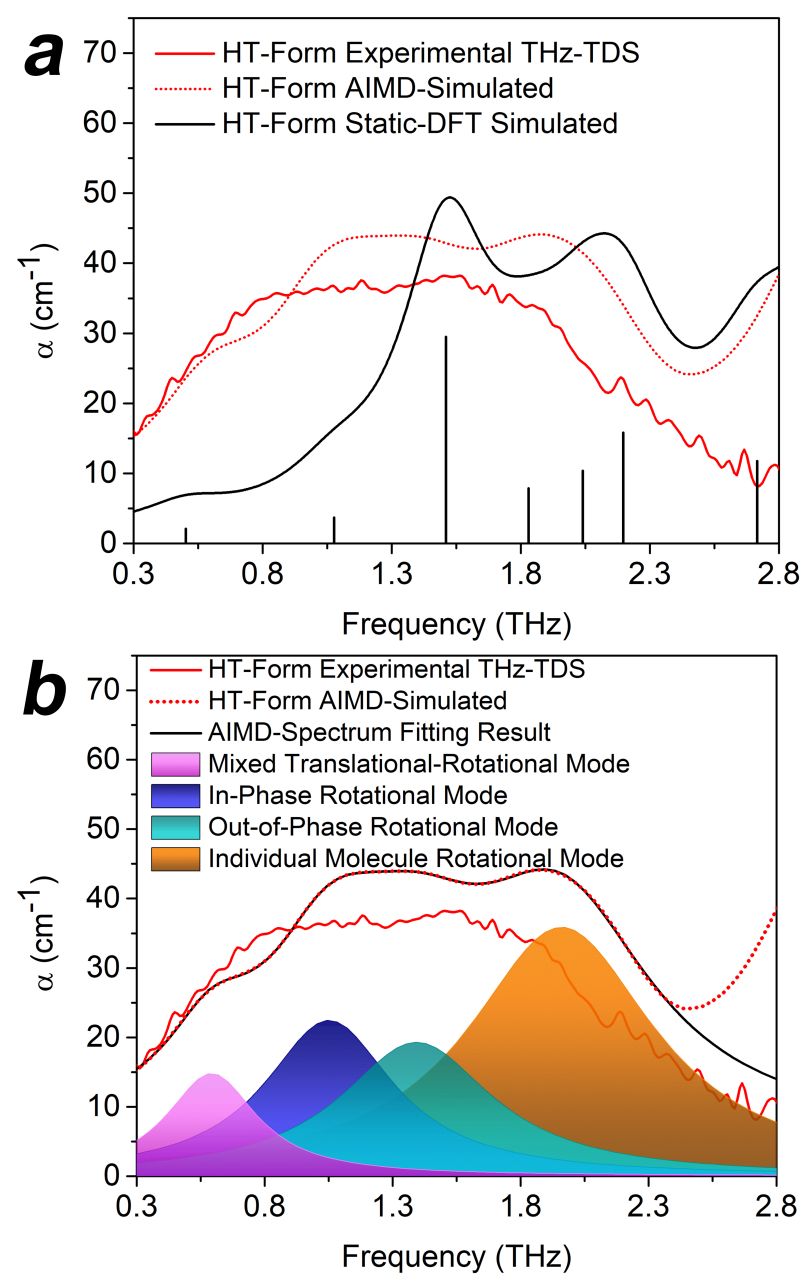

FIG. 3: (a) Comparison between the experimental THz-TDS spectrum (red, solid), AIMD-simulated (red, dotted), and static-DFT simulated (black) spectra of the HT-phase of camphor, and (b) the result of fitting the AIMD-simulated spectrum (black) with four Lorentzian curves using the static-DFT frequencies as the basis for the fit. The average of the residual over the fitted range $(0.3-2.3 \mathrm{THz})$ is only 0.09 , highlighting the excellent fit achieved.

shifts to higher energies (from $0.503 \mathrm{THz}$ to $0.5802 \mathrm{THz}$ ). Because this mode represents the phase-transformation pathway, this indicates that the vibrational potential is stiffening with increasing temperature hence making it more energetically unfavourable to revert back to the LT-phase. This could help explain why at lower temperatures, as well as when performing unconstrained optimisations, the system tends to revert back to the LT-form, highlighting the overall importance of this particular vibration on the order-disorder transition in camphor.

The understanding gained through these combined methods makes it possible to develop deeper insight into the phase-transition processes, specifically related to the 
kinetics and thermodynamics of the transition. As previously reported, the phase transition of camphor is highly sensitive to both temperature and pressure, and the transition exhibits a hysteresis during subsequent heating and cooling cycles. The comparison between the experimental phase transition temperature and pressures and the static-DFT transition points, obtained by calculating the pressure and temperature dependent free energies of the two forms and determining the crossing points, are shown in Figure 4. Overall, they are in good agreement with each other and The lack in complete quantitative agreement originates from inherent limitations of the technique, i.e. not taking into account thermal expansion or anharmonic effects, and can be considerably improved by performing quasi-harmonic approximation (QHA) simulations, $[1,41]$ which aim to take thermal expansion effects into account that. Additionally, the DFTresults enable more specific details surrounding the thermodynamic driving force to be determined, and the results show that at the phase transition the HT-form in entropically favoured by $9.32 \mathrm{~J} \mathrm{~mol}^{-1} \mathrm{~K}^{-1}$, further indicating the importance of the low-frequency phonons on the phase transformation behaviour. While the staticDFT method is unable to directly capture the hysteresis behaviour due to inherent limitations in the technique (i.e. only dependent on the final two states), the general agreement provides another level of validation that both the theoretical model used, as well as the generated structure, are accurate.

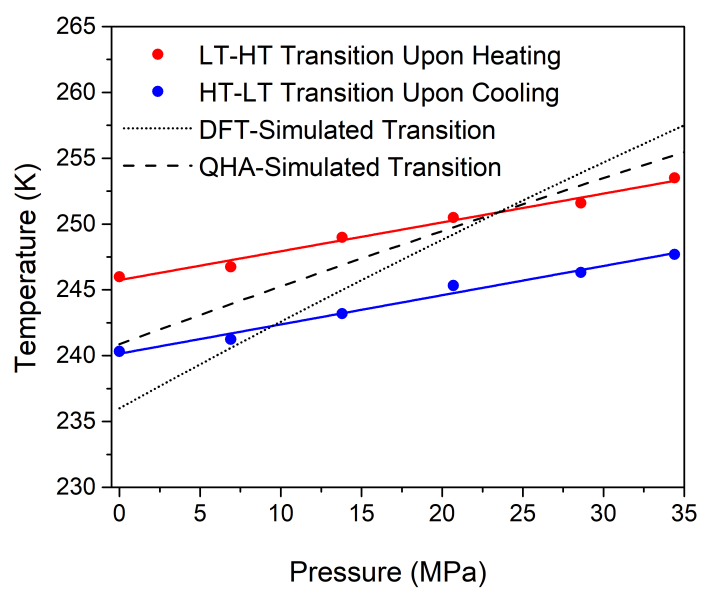

FIG. 4: The experimentally observed phase transition from THz-TDS as a function of temperature and pressure for the LT-HT (blue) and HT-LT (red)

transformation. The dotted and dashed lines are the predicted transition points determined from the static-DFT and QHA vibrational simulations, respectively.

The experimental observation of a hysteresis is likely due to two complementary processes: the restricted dy- namics at lower temperatures that hinder reorganisation into a coherent crystalline solid, and the potential energy barriers associated with the rearrangement from the disordered state. Combined, these lead to significant kinetic constraints (i.e. significant molecular mobility to undergo crystal nucleation), and slight thermodynamic (free-energy) barriers, that make the formation of a crystalline solid less likely without a significant energetic gradient, which occurs at lower-temperatures. By performing $5 \mathrm{ps}$ NPT simulations starting from the LT and HT-phases at temperatures above and below the transition temperature, respectively, the rates of the $\gamma$ angle changes were extracted. These gradients $\left(\approx 1.5\right.$ and $0.2^{\circ} \mathrm{ps}^{-1}$ for the LT to HT and HT to LT transitions, respectively) were used to determine a temperature-dependent rate constant for the two transformations, and the collision frequencies and activation energies of the two transitions can be extracted (see Supporting Information for more details). The calculated prefactors have values of 1.91 and $0.57 \mathrm{THz}$ for the LTHT and HT-LT transitions, respectively, which are in good agreement with the observed vibrational modes that correspond to the phase-transformation pathway. The results also indicate that the barrier of the LT-HT transition is lower in energy compared to the barrier of the HTLT transition by approximately $245 \pm 61 \mathrm{~J} \mathrm{~mol}^{-1}$, which is corresponds to a temperature of $30 \mathrm{~K}$. While the experimentally determined hysteresis exhibits approximately $5 \mathrm{~K}$ width, the relative agreement represents a significant achievement of the theoretical methodology, which is capable of fully interpreting the mechanistic and energetic origins of the observed behavior. Moreover, this is well below (almost 20 times lower than) the $1 \mathrm{kcal} \mathrm{mol}^{-1}$ $\left(4.2 \mathrm{~kJ} \mathrm{~mol}^{-1}\right)$ 'chemical-accuracy' standard that is often referred to in the theoretical chemistry community.[42]

M.T.R. and J.A.Z. thank the UK Engineering and Physical Sciences Research Council for funding (EP/N022769/1) and the ARCHER National HPC for resources. W.Z. and D.M.M. thank the R. A. Welch Foundation for funding. Additional data related to this publication are available at the University of Cambridge data repository (https://doi.org/10.17863/CAM.21664).

* mtr34@cam.ac.uk

[1] M. T. Ruggiero, J. A. Zeitler, and A. Erba, Chem. Commun. 53, 3781 (2017).

[2] M. T. Ruggiero, J. Sibik, R. Orlando, J. A. Zeitler, and T. M. Korter, Angew. Chem. Int. Ed. 55, 6877 (2016).

[3] D. a. Turton, H. M. Senn, T. Harwood, A. J. Lapthorn, E. M. Ellis, and K. Wynne, Nat. Commun. 5, 028103 (2014).

[4] J. M. Pecourt, J. Peon, and B. Kohler, J. Am. Chem. Soc. 123, 10370 (2001).

[5] M. González-jiménez, G. Ramakrishnan, T. Harwood, 
A. J. Lapthorn, S. M. Kelly, E. M. Ellis, and K. Wynne, Nat. Commun. 7, 11799 (2016).

[6] S. Illig, A. S. Eggeman, A. Troisi, L. Jiang, C. Warwick, M. Nikolka, G. Schweicher, S. G. Yeates, Y. H. Geerts, J. E. Anthony, and H. Sirringhaus, Nat. Commun. 7, 10736 (2016).

[7] T. Tanno, Y. Watanabe, K. Umeno, A. Matsuoka, H. Matsumura, M. Odaka, and N. Ogawa, J. Phys. Chem. C 121, 17921-17924 (2017).

[8] N. Y. Tan, M. T. Ruggiero, C. Orellana-tavra, T. Tian, A. D. Bond, T. M. Korter, D. Fairen-jimenez, and J. Axel Zeitler, Chem. Commun. 51, 16037 (2015).

[9] E. P. J. Parrott and J. A. Zeitler, Appl. Spectrosc. 69, 1 (2014).

[10] G. J. O. Beran, Chem. Rev. 116, 5567 (2016).

[11] J. Sibik and J. A. Zeitler, Philos. Mag. 96, 842 (2015).

[12] L. Genberg, R. J. D. Miller, F. Heisel, and G. Mclendon, J. Phys. Chem. 91, 5521 (1987).

[13] J. Sibik, K. Löbmann, T. Rades, and J. A. Zeitler, Mol. Pharmaceutics 12, 3062 (2015).

[14] Veaceslav Coropceanu, Jérôme Cornil, Demetrio A da Silva Filho, Yoann Olivier, R. Silbey, , and Jean-Luc Brédas, Chem. Rev. 107, 926 (2007).

[15] S. Ebbinghaus, S. J. Kim, M. Heyden, X. Yu, U. Heugen, M. Gruebele, D. M. Leitner, and M. Havenith, Proc. Natl. Acad. Sci. U.S.A. 104, 20749 (2007).

[16] H. Hoshina, K. Kawase, and A. Irizawa, Sci. Rep. 6, 27180 (2016)

[17] C. C. Mjojo, J. Chem. Soc., Faraday Trans. 2 75, 692 (1979).

[18] J. E. Anderson and W. P. Slichter, J. Chem. Phys. 41, 1922 (1964).

[19] M. Brunelli, A. N. Fitchci, and A. J. Mora, J. Solid State Chem. 163, 253 (2002).

[20] D. V. Nickel, M. T. Ruggiero, T. M. Korter, and D. M. Mittleman, Phys. Chem. Chem. Phys. 17, 6734 (2015).

[21] W. Zhang, D. Nickel, and D. Mittleman, Opt. Express 25, 2983 (2017).

[22] J. R. Hutter, J. Hutter, J. Vandevondele, M. Iannuzzi, M. Iannuzzi, J. Vandevondele, F. Schiffmann, F. Schiffmann, and J. Vandevondele, WIREs Comput Mol Sci 4, 15 (2013).

[23] J. Vandevondele, M. Krack, F. Mohamed, M. Parrinello, T. Chassaing, and J. Hutter, Comput. Phys. Commun. 167, 103 (2005).

[24] S. Grimme, J. Antony, S. Ehrlich, and H. Krieg, J. Chem. Phys. 132, 154104 (2010).
[25] S. Grimme, S. Ehrlich, and L. Goerigk, J. Comput. Chem. 32, 1456 (2011).

[26] J. Perdew, K. Burke, and M. Ernzerhof, Phys. Rev. Lett. $\mathbf{7 7}, 3865$ (1996).

[27] J. Vandevondele and J. Hutter, J. Chem. Phys. 127, 114105 (2007).

[28] S. Goedecker, M. Teter, and J. Hutter, Phys. Rev. B (1996).

[29] S. Nosé, J. Chem. Phys. 81, 511 (1998).

[30] G. J. Martyna, D. J. Tobias, and M. L. Klein, J. Chem. Phys. 101, 4177 (1994).

[31] G. J. Martyna, M. L. Klein, and M. Tuckerman, J. Chem. Phys. 97, 2635 (1992).

[32] M. T. Ruggiero, M. Krynski, E. O. Kissi, J. Sibik, D. Markl, N. Y. Tan, D. Arslanov, W. Van Der Zande, B. Redlich, T. M. Korter, H. Grohganz, K. Lobmann, T. Rades, S. R. Elliott, and J. A. Zeitler, Phys. Chem. Chem. Phys. 19, 30039 (2017).

[33] R. Dovesi, R. Orlando, A. Erba, C. M. Zicovich-wilson, B. Civalleri, S. Casassa, L. Maschio, M. Ferrabone, M. De La Pierre, P. D'arco, Y. Noël, M. Causà, M. Rérat, and B. Kirtman, Int. J. Quantum Chem. 114, 1287 (2014).

[34] F. Weigend and R. Ahlrichs, Phys. Chem. Chem. Phys. 7, 3297 (2005).

[35] F. Affouard, J. F. Willart, and M. Descamps, in J. NonCryst. Solids 307-310, 9-15 (2002) .

[36] A. Hédoux, Y. Guinet, P. Derollez, J.-F. Willart, F. Capet, and M. Descamps, J. Phys. Condens. Matter 15, 8647 (2003).

[37] M. Brehm and B. Kirchner, J. Chem. Inf. Model. 51, 2007 (2011).

[38] M. T. Ruggiero and J. A. Zeitler, J. Phys. Chem. B 120, 11733 (2016).

[39] F. Pascale, C. M. Zicovich-wilson, F. L. Gejo, B. Civalleri, R. Orlando, and R. Dovesi, J. Comput. Chem. 25, 888 (2004).

[40] Y. Noël, C. Zicovich-wilson, C. M. Zicovich-wilson, R. Dovesi, B. Civalleri, P. D'arco, and R. Dovesi, Phys. Rev. B 65, 014111 (2001).

[41] A. Erba, J. Maul, and B. Civalleri, Chem. Commun. 52, 1820 (2016).

[42] J. Brandenburg and S. Grimme, in Prediction and Calculation of Crystal Structures, Topics in Current Chemistry, Vol. 345, edited by S. Atahan-Evrenk and A. AspuruGuzik (Springer International Publishing, 2014) pp. 123. 